\title{
Política inclusiva del tercer ciclo de la educación escolar básica del centro regional de educación pilar, año 2020-2021
}

\author{
Liz Maira Galeano Pino \\ lizgaleano15.lg@gmail.com \\ Maestría en Ciencias de la Educación, \\ con énfasis en Investigación Científica. \\ Facultad de Humanidades y Ciencias de la Educación \\ Universidad Nacional de Pilar
}

\section{RESUMEN}

Con este trabajo se analiza la manera que se realiza la atención a los alumnos con necesidades específicas de apoyo educativo en el Tercer Ciclo de la Educación Escolar Básica del Centro Regional de Educación “Mariscal Francisco Solano López” Pilar, año 2020-2021. La investigación está basada en los métodos cualitativo y cuantitativo que permitirá aportar informaciones y datos relacionados a la política institucional en materia de inclusión educativa, así como las acciones del equipo técnico y estrategias utilizadas por los docentes en el aula, y el acompañamiento de los padres a estos alumnos. Se ha llegado a la conclusión que se ha alcanzado un nivel máximo del (Nivel 3-Simpre) donde los profesionales de la educación son partidarios y 'participes de la inclusión educativa, manifiestan buena actitud y expectativas positivas hacia sus alumnos, independientemente de cuáles sean sus características, reflejando en las dimensiones cultura, políticas y prácticas han alcanzado valoraciones muy elevada se relaciona con la preparación necesaria y específica para el desarrollo de prácticas inclusivas, al destacar la importancia de crear una cultura escolar en la que los estudiantes se sientan competentes, valorados y no excluidos. Para que el derecho a la educación sea garantizado con justicia éste tiene que ser reconocido y aplicado a todas las personas, sin excepción alguna, siendo obligación del Estado tomar las medidas necesarias para proteger al niño de toda forma de discriminación.

Palabras Claves: atención a los alumnos; necesidades específicas; apoyo educativo. 


\title{
Inclusive policy of the third cycle of basic school education of the regional center of Pilar education, year 2020-2021
}

\begin{abstract}
This work analyzes how the attention to students with specific educational support needs is carried out in the Third Cycle of Basic School Education of the Regional Center for Education "Mariscal Francisco Solano López" Pilar, year 2020-2021. The research is based on qualitative and quantitative methods that will provide information and data related to the institutional policy on educational inclusion, as well as the actions of the technical team and strategies used by teachers in the classroom, and the accompaniment of parents. these students. It has been concluded that a maximum level of (Level 3-Simpre) has been reached where education professionals are supporters and 'participants of educational inclusion, they manifest a good attitude and positive expectations towards their students, regardless of what they are. Its characteristics, reflecting in the dimensions culture, policies and practices, have reached very high valuations, it is related to the necessary and specific preparation for the development of inclusive practices, highlighting the importance of creating a school culture in which students feel competent, valued and not excluded. For the right to education to be justly guaranteed, it must be recognized and applied to all people, without exception, and the State is obliged to take the necessary measures to protect the child from all forms of discrimination.
\end{abstract}

Keywords: attention to students; specific needs; educational support.

Artículo recibido: 15 octubre. 2021 Aceptado para publicación: 18 noviembre 2021 Correspondencia: $\underline{\text { lizgaleano15.lg@ gmail.com }}$ Conflictos de Interés: Ninguna que declarar 


\section{INTRODUCCIÓN}

La UNESCO define la educación inclusiva como un proceso orientado a responder a la diversidad de los estudiantes incrementando su participación y reduciendo la exclusión en y desde la educación. Está relacionada con la presencia, la participación y los logros de todos los alumnos, con especial énfasis en aquellos que, por diferentes razones, están excluidos o en riesgo de ser marginados, constituyendo un impulso fundamental para avanzar en la agenda de la Educación para Todos (Organización de las Naciones Unidas para la Educación, la Ciencias y la Cultura, 2008).

En tal sentido, día a día el Ministerio de Educación y Ciencias presenta resoluciones relacionadas a la atención de alumnos con necesidades específicas de apoyo educativo, considerando las necesidades derivadas de discapacidad, trastornos específicos de aprendizaje, altas capacidades intelectuales, incorporación tardía al sistema educativo, condiciones personales o de historia escolar, requiera de apoyos o ajustes para alcanzar el máximo desarrollo posible de sus capacidades personales.

La educación inclusiva es, casi con toda seguridad, uno de los "temas estrella" o asuntos de interés que ocupan y preocupan las agendas de la política educativa latinoamericana en la actualidad. Es este un concepto relativamente reciente, pero que en el corto devenir de los últimos años ha sufrido serias transformaciones, ampliando su entidad y significatividad, recogiendo o englobando en el mismo, a términos más familiares en el área de la política educativa como la calidad, la equidad, el acceso, la diversidad, la participación, etc (Rico, 2010).

Según las políticas educativas inclusivas en América Latina en cuanto a los problemas y desafíos, la calidad, la equidad y, en menor medida, la inclusión son los tres conceptos que orientan las políticas educativas en América Latina. Los países implementan numerosas políticas, planes y programas con dos enfoques complementarios: estrategias unitarias e integrales de apoyo y acompañamiento educativo; y acciones redistributivas y afirmativas para grupos vulnerables para luchar contra las causas de exclusión. Aunque, generalmente, no existen estrategias unificadas y enfoques integrados, sin políticas coordinadas de lucha contra la exclusión y la desigualdad (Rico, 2010).

Es por ello que se necesita conocer la realidad local en materia de inclusión, saber cómo se atiende a los alumnos que requieren de políticas educativas inclusivas y analizar cómo se realizan las a acciones que beneficien a los mismos, con el fin de crear 
protocolos de trabajo y concienciar a docentes y padres a trabajar unidos en busca de una sociedad más inclusiva.

La educación inclusiva es el proceso sistémico de mejora e innovación educativa para promover la presencia, el rendimiento y la participación del alumnado en todas las instituciones del sistema educativo nacional donde son escolarizados, con particular atención a aquellos alumnos o alumnas frente más vulnerables a la exclusión, el fracaso escolar o la marginación, detectando y eliminando, para ello, las barreras que limitan dicho proceso (Ley No5136 Educación Inclusiva, 2013), atendiendo lo citado, la escuela debe fomentar la educación inclusiva y potenciar las capacidades que tenga los alumnos con necesidades específicas de apoyo educativo haciendo que los mismos adquieran experiencias según sus necesidades, considerando el tiempo, el grado y las características del proceso de enseñanza.

El niño y el adolescente con discapacidad física, sensorial, intelectual o emocional, tienen derecho a recibir cuidados y atención adecuados, inmediatos y continuos, que contemplen estimulación temprana y tratamiento educativo especializado, tendiente a su rehabilitación e integración social y laboral, que le permitan valerse por sí mismos y participar de la vida de su comunidad en condiciones de dignidad e igualdad. En ningún caso se permitirá la discriminación o el aislamiento social de los afectados" (Código de la Niñez y la Adolescencia,1998), basándonos en lo citado, la escuela debe fomentar la educación inclusiva y potenciar las capacidades que tenga los alumnos con necesidades específicas de apoyo educativo haciendo que los mismos adquieran experiencias según sus necesidades, considerando el tiempo, el grado y las características del proceso de enseñanza.

A través del presente trabajo se determina la Política Inclusiva del Tercer Ciclo de la Educación Escolar Básica del Centro Regional de Educación "Mariscal Francisco Solano López" Pilar - Paraguay, año 2020-2021, de manera a recabar datos de la forma de trabajo en el entorno y la posibilidad de crear protocolos basados en experiencias concretas.

\section{REVISION BIBLOGRÁFICA}

\section{Los alumnos con necesidades específicas de apoyo educativo:}

De acuerdo a la Ley No5136 de Educación Inclusiva, (2013). Se considera a todo alumno que debido a: necesidades específicas de apoyo educativo: derivadas de 
discapacidad física, intelectual auditiva, visual y psicosocial, trastornos específicos de aprendizaje, altas capacidades intelectuales, incorporación tardía al sistema educativo, condiciones personales o de historia escolar, requiera de apoyos y/o ajustes para alcanzar el máximo desarrollo posible de sus capacidades personales.

La discapacidad es una condición o situación por la cual una persona, con alguna deficiencia y con un entorno inapropiado por los diversos obstáculos y falta de apoyos necesarios, no puede realizar ciertas actividades o no puede "funcionar" en algunas cosas como otras personas de su edad. (Ley N5136 Educación Inclusiva, 2013)

La discapacidad es el resultado de la relación entre un apersona con limitaciones o deficiencias y los obstáculos del medio que no le permiten participar en la vida social como otras personas. (Convención Internacional por los Derechos de las Personas con Discapacidad de Naciones Unidas, aprobada en Paraguay por la Ley No 3540/08)

La discapacidad no es una enfermedad, es una característica de las personas. El término adecuado para referirse a este grupo de la población es Persona con Discapacidad (PcD), según lo establecido en el Marco Legal que Promueve la Inclusión de Personas con Discapacidad, elaborado por Fundación Saraki.

Los trastornos específicos de aprendizaje constituyen un conjunto de problemas que interfieren significativamente en el rendimiento en la escuela, dificultando el adecuado progreso del niño y la consecución de las metas marcadas en los distintos planes educativos, y que de acuerdo a la Ley requieren ajustes razonables que son las modificaciones y adaptaciones necesarias y adecuadas que no impongan una carga desproporcionada o indebida, cuando se requieran en un caso particular, para garantizar a los alumnos con necesidades específicas de apoyo educativo el goce o ejercicio, en igualdad de condiciones con los demás de los derechos humanos y libertades fundamentales (Ley N5136 Educación Inclusiva, 2013).

\section{Los niveles de iniquidad, exclusión y fragmentación social.}

Pese a la gran expansión de la educación y los esfuerzos realizados, todavía persisten desigualdades educativas en función de los distintos estratos socioeconómicos, culturas y características individuales del alumnado, como consecuencia del modelo homogeneizador de los sistemas educativos. Una mayor equidad es un factor fundamental para conseguir un mayor desarrollo, una integración social y una cultura de la paz basada en el respeto y valoración de las diferencias. Por ello, es preciso avanzar 
hacia el desarrollo de escuelas más inclusivas que eduquen en la diversidad y que entiendan ésta como una fuente de enriquecimiento para el aprendizaje y los procesos de enseñanza (Vaughan, 2002, p.7).

El principio rector del Marco de Acción de la Conferencia Mundial sobre necesidades especiales (Salamanca, 1994) es que todas las escuelas deben acoger a todos los niños independientemente de sus condiciones personales, culturales o sociales; niños discapacitados y bien dotados, niños de la calle, de minorías étnicas, lingüísticas o culturales, de zonas desfavorecidas o marginales, lo cual plantea un reto importante para los sistemas educativos. La inclusión no tiene que ver sólo con el acceso de los alumnos y alumnas con discapacidad a las escuelas comunes, sino con eliminar o minimizar las barreras que limitan el aprendizaje y la participación de todo el alumnado (Vaughan, 2002, p.7).

Muchos estudiantes experimentan dificultades porque no se tienen en cuenta sus diferencias en los procesos de enseñanza y aprendizaje. Los diversos grupos sociales, etnias y culturas tienen normas, valores, creencias y comportamientos distintos, que generalmente no forman parte de la cultura escolar, lo que puede limitar sus posibilidades de aprendizaje y de participación, o conducir a la exclusión y discriminación. La oferta curricular, la gestión escolar, las estrategias de aprendizaje que se utilizan en el aula y las expectativas de los profesores, entre otros, son factores que pueden favorecer o dificultar el desarrollo y aprendizaje de los alumnos y su participación en el proceso educativo. El mismo alumno puede tener dificultades en una escuela y no en otra, dependiendo de cómo se aborden en cada una las diferencias. Esto significa que, si la escuela puede generar dificultades, también está en su mano poder evitarlas. La escuela tiene, por tanto, un papel fundamental para evitar que las diferencias de cualquier tipo se conviertan en desigualdades educativas y por esa vía en desigualdades sociales, produciéndose un círculo vicioso difícil de romper. Las escuelas inclusivas representan un marco favorable para asegurar la igualdad de oportunidades y la plena participación, contribuyen a una educación más personalizada, fomentan la colaboración entre todos los miembros de la comunidad escolar y constituyen un paso esencial para avanzar hacia sociedades más inclusivas y democráticas (Vaughan, 2002, p.7). 
Muchas escuelas de América Latina han iniciado el camino de la inclusión o están interesadas en hacerlo, sin embargo, pueden sentirse perdidas o tener dudas respecto de cómo avanzar hacia una mayor inclusión (Vaughan, 2002, p.7).

\section{Barreras para el aprendizaje y la participación}

Las barreras para el aprendizaje y la participación son obstáculos de índole arquitectónico, comunicacional, metodológico, instrumental, programático, actitudinal y tecnológico que dificultan o inhiben las posibilidades de aprendizaje de los alumnos con necesidades específicas de apoyo educativo (Ley N5136 Educación Inclusiva, 2013)

Por lo que se requiere la inclusión, la identificación y minimización de las barreras para el aprendizaje y la participación, y maximización de los recursos para el apoyo de ambos procesos (Ley №5136 Educación Inclusiva, 2013).

La Educación inclusiva son procesos sistémicos de mejora e innovación educativa para promover la presencia, el rendimiento y la participación del alumnado en todas las instituciones del sistema educativo nacional donde son escolarizados, con particular atención a aquellos alumnos o alumnas frente más vulnerables a la exclusión, el fracaso escolar o la marginación, detectando y eliminando, para ello, las barreras que limitan dicho proceso (Ley No5136 Educación Inclusiva, 2013).

La inclusión demanda una nueva manera de pensar sobre las diferencias y la diversidad, percibiéndola como algo valioso en sí mismo que requiere un procedimiento educativo y social apropiado (Escribano y Martínez, 2013).

"La educación inclusiva es, ante todo y en primer lugar, una cuestión de derechos humanos, ya que define que no se puede segregar a ninguna persona como consecuencia de su discapacidad o dificultad de aprendizaje, género o pertenencia a una minoría étnica" (Arnaiz Sánchez, 2003).

Hablar de inclusión no es una simple cuestión curricular, organizativa o metodológica, ella, es más que todo eso, es una manera distinta de encarar la educación, con humanismo, con un cumulo de valores que se han vistos ocultos en los últimos años.

El modelo de escuela inclusiva se presenta hoy como el camino hacia donde deben dirigirse los esfuerzos de cambio que exige el sistemas educativo de nuestro país, tratando de ofrecer una educación integral y de calidad a todos los alumnos, sin importar sus características individuales, sino el desarrollo máximo sus capacidades, 
garantizando la diversidad y la igualdad de oportunidades. Y este camino se hará más corto a medida que tomemos en cuenta las recomendaciones de quienes han tenido experiencias enriquecedoras en este ámbito.

Una institución inclusiva es aquella, que ofrece a todos sus alumnos las mismas oportunidades educativas y la atención necesaria para el progreso académico y personal de todos.

En inclusión, siempre se debe considerar el principio de que cada alumno tiene sus propias características, intereses, capacidades y necesidades de aprendizaje distintos, que deben ser atendidos tal y como lo pide los fundamentos y principios de la educación inclusiva.

El sistema educativo actual presenta adecuaciones ajustadas a la necesidad de inclusión, que deben ser atendidas por todos los actores educativo (docentes, directivos, equipo técnico institucional, supervisores, miembros de entres gubernamentales y sociedad civil)

Según el informe de la UNESCO, (1996), "la educación puede ser un factor de cohesión social si procura tener en cuenta la diversidad de los individuos y de los grupos humanos y al mismo tiempo evita ser a su vez un factor de exclusión social" (Sánchez Palomino, 2009).

Según lo citado en el Código de la Niñez y la Adolescencia, (1998). "El niño y el adolescente con discapacidad física, sensorial, intelectual o emocional, tienen derecho a recibir cuidados y atención adecuados, inmediatos y continuos, que contemplen estimulación temprana y tratamiento educativo especializado, tendiente a su rehabilitación e integración social y laboral, que le permitan valerse por sí mismos y participar de la vida de su comunidad en condiciones de dignidad e igualdad. En ningún caso se permitirá la discriminación o el aislamiento social de los afectados", por lo que la escuela debe fomentar la educación inclusiva y potenciar las capacidades que tenga los alumnos con necesidades específicas de apoyo educativo haciendo que los mismos adquieran experiencias según sus necesidades, considerando el tiempo, el grado y las características del proceso de enseñanza.

Las escuelas deben buscar los mecanismos para atender a todos los alumnos por igual y considerar que muchos necesitan de una enseñanza más individualizada para el 
desarrollo de sus capacidades, garantizando el acceso y la promoción educativa de los mismos, con una adecuada atención y estimulación.

El proceso de evaluación de los alumnos con necesidades específicas de apoyo educativo será responsabilidad del equipo técnico institucional con la participación del docente y tendrá por finalidad determinar el nivel de competencia curricular, definir los ajustes razonables individuales y los requerimientos de apoyo. Para la implementación de ajustes razonables, se requerirá en cada caso particular una evaluación cualitativa, cuantitativa y diagnóstica. Asimismo, se deberán promover acciones para la remoción de barreras para el aprendizaje y la participación. Los ajustes razonables aplicados individualmente quedarán registrados en el expediente de los alumnos. Este será la referencia principal para su evaluación y promoción (Ley No 5136/13 De Educación Inclusiva, 2013).

En caso de que los ajustes razonables individuales se distancien significativamente de los aprendizajes establecidos en el currículum regular, se flexibilizará la oferta curricular y se darán los apoyos necesarios de modo a que se asegure la permanencia del alumno/a en la institución educativa, así como su promoción (Ley $N^{\text {o }}$ 5136/13 De Educación Inclusiva, 2013).

Los criterios para la evaluación de los aprendizajes de los alumnos con necesidades específicas de apoyo educativo, que hayan requerido ajustes razonables, se definirán de acuerdo con los objetivos o contenidos propuestos para cada caso en particular. Para establecer los resultados de la evaluación administrada a los alumnos con necesidades específicas de apoyo educativo, se deberá aplicar la misma escala de calificación dispuesta en la normativa de evaluación vigente. Asimismo, se les entregará el boletín de calificaciones en las mismas condiciones que los demás alumnos, con la aclaración que han sido evaluados conforme a las adecuaciones ajustadas a sus necesidades.

Los alumnos, con necesidades específicas de apoyo educativo serán promovidos de grado y deberán recibir las certificaciones correspondientes. (Ley No 5136/13 De Educación Inclusiva, 2013).

Los alumnos, con necesidades específicas de apoyo educativo que hayan logrado los objetivos establecidos obtendrán una certificación y deberá garantizarse su continuidad y permanencia en el servicio educativo. A aquellos que demuestren competencias curriculares superiores a las que correspondan a su grupo de edad, se les ofrecerán 
opciones curriculares adecuadas a su nivel de habilidades y conocimientos (Ley $\mathrm{N}^{\mathrm{o}}$ 5136/13 De Educación Inclusiva, 2013).

\section{Las Dimensiones inclusivas.}

\section{- La Dimensión A: Crear CULTURAS Inclusivas.}

Esta dimensión se relaciona con la creación de una comunidad escolar segura, acogedora, colaboradora y estimulante, en la que cada uno es valorado, lo cual es la base fundamental primordial para que todo el alumnado tenga mayores niveles de logro. Se refiere, asimismo, al desarrollo de valores inclusivos, compartidos por todo el personal de la escuela, los estudiantes, los miembros del Consejo Escolar y las familias, que se transmitan a todos los nuevos miembros de la comunidad escolar. Los principios que se derivan de esta cultura escolar son los que guían las decisiones que se concretan en las políticas escolares de cada escuela y en su quehacer diario, para apoyar el aprendizaje de todos a través de un proceso continuo de innovación y desarrollo de la escuela (Vaughan, 2002, p.7).

\section{- La Dimensión B: Elaborar POLÍTICAS inclusivas}

Esta dimensión tiene que ver con asegurar que la inclusión sea el centro del desarrollo de la escuela, permeando todas las políticas, para que mejore el aprendizaje y la participación de todo el alumnado. Se considera como "apoyo" todas las actividades que aumentan la capacidad de una escuela para dar respuesta a la diversidad del alumnado. Todas las modalidades de apoyo se agrupan dentro de un único marco y se conciben desde la perspectiva del desarrollo de los alumnos, y no desde la perspectiva de la escuela o de las estructuras administrativas (Vaughan, 2002, p.7).

\section{- La Dimensión C: Desarrollar PRÁCTICAS inclusivas}

Esta dimensión se refiere a que las prácticas educativas reflejen la cultura y las políticas inclusivas de la escuela. Tiene que ver con asegurar que las actividades en el aula y las actividades extraescolares promuevan la participación de todo el alumnado y tengan en cuenta el conocimiento y la experiencia adquiridos por los estudiantes fuera de la escuela. La enseñanza y los apoyos se integran para "orquestar" el aprendizaje y superar las barreras al aprendizaje y la participación. El personal moviliza recursos de la escuela y de las instituciones de la comunidad para mantener el aprendizaje activo de todos (Vaughan, 2002, p.7). 
Estas dimensiones se han elegido para orientar la reflexión hacia los cambios que se deberían llevar a cabo en las escuelas. Durante muchos años, se ha prestado poca atención al potencial de la cultura de las escuelas, siendo éste un factor importante que puede facilitar o limitar el aprendizaje y los cambios en la enseñanza. A través del desarrollo de culturas inclusivas se pueden producir cambios en las políticas y en las prácticas, que pueden mantenerse y transmitirse a los nuevos miembros de la comunidad escolar. Aunque estas tres dimensiones aparecen en un determinado orden en este documento, deben ser consideradas de igual importancia para el desarrollo de la inclusión en la escuela. Se pueden representar como los lados de un triángulo equilátero (Vaughan, 2002, p.7).

Cada dimensión se divide en dos secciones, que centran la atención en un conjunto de actividades en las que deben comprometerse las escuelas, como una vía para ir mejorando el aprendizaje y la participación de todo el alumnado.

\section{Dimensión A: Crear CULTURAS inclusivas}

1. Construir una comunidad

2. Establecer valores inclusivos

\section{Dimensión B: Elaborar POLÍTICAS inclusivas}

1. Desarrollar una escuela para todos

2. Organizar el apoyo para atender a la diversidad

\section{Dimensión C: Desarrollar PRÁCTICAS inclusivas}

1. Orquestar el proceso de aprendizaje

2. Movilizar recursos

Estas dimensiones y secciones también se pueden utilizar para estructurar el plan de desarrollo de la escuela y servir de epígrafes principales del mismo. Si el significado del encabezamiento de una determinada sección no se comprende de forma inmediata, éste se puede clarificar volviendo a leer la breve descripción que se ha hecho anteriormente de las dimensiones. Cada sección contiene un conjunto de indicadores, doce como máximo, y el significado de cada uno de ellos se clarifica a través de una serie de preguntas. Las dimensiones, las secciones, los indicadores y las preguntas proporcionan un mapa cada vez más detallado que guía el análisis de la situación de la escuela en ese momento y determina futuras posibilidades de acción (Vaughan, 2002, p.8). 
Es importante recordar que las dimensiones se superponen entre sí, ya que los avances en la cultura escolar requieren, a su vez, la formulación de políticas y la implementación de prácticas.

Los indicadores representan una declaración de "aspiraciones" con las que se compara la situación existente en la escuela a fin de establecer determinadas prioridades de desarrollo. En algunas escuelas, el personal y los miembros del Consejo Escolar pueden concluir que no desean comprometerse con determinados indicadores, por el momento, o que éstos no indican la dirección hacia la que desean avanzar (Vaughan, 2002, p.8).

La inclusión en educación implica procesos para aumentar la participación de los estudiantes y para reducir su exclusión, en la cultura, los currícula y las comunidades de las escuelas.

- La inclusión implica reestructurar la cultura, las políticas y las prácticas de los centros educativos para que puedan atender la diversidad del alumnado de su localidad.

- La inclusión se refiere al aprendizaje y la participación de todos los estudiantes vulnerables de ser sujetos de exclusión, no sólo aquellos con discapacidad o etiquetados como “con Necesidades Educativas Especiales".

- La inclusión se refiere al desarrollo de las escuelas tanto del personal como del alumnado. (Vaughan, 2002, p.8).

- La preocupación por superar las barreras para el acceso y la participación de un alumno en particular puede servir para revelar las limitaciones más generales de la escuela a la hora de atender a la diversidad de su alumnado.

- Todos los estudiantes tienen derecho a una educación en su localidad.

- La diversidad no se percibe como un problema a resolver, sino como una riqueza para apoyar el aprendizaje de todos.

- La inclusión se refiere al refuerzo mutuo de las relaciones entre los centros escolares y sus comunidades.

- La inclusión en educación es un aspecto de la inclusión en la sociedad (Vaughan, 2002, p.8).

\section{Prácticas pedagógicas inclusivas}

Según (Carrillo. et.al, 2018, p.3). Las prácticas pedagógicas son una de las partes más importantes dentro del que hacer educativo de los agentes que manejaran o manejan las 
aulas inclusivas ya que como lo describen anteriormente Agudelo y colaboradores son las prácticas situadas dentro del contexto educativo y social y son las que dan un eje centrar a la educación ya que de ahí se implementarán los pasos a seguir en las aulas y cómo se dirigirá las prácticas ya establecidas y se trasformará a un nuevo paradigma de inclusión educativa ya que se busca conocer las prácticas actuales bajo una luz de inclusión. Según Ainscow (2017) estas prácticas aportan a la dinamización de los recursos disponibles con el fin de cubrir las necesidades básicas y especiales de la enseñanza y el aprendizaje. Desde la perspectiva del autor López (2011) dentro de las escuelas es necesario dejar de hablar de las barreras en el aprendizaje de los niños y niñas con algún tipo de discapacidad, en vez de eso se debe priorizar en las estrategias y prácticas que los docentes implementen en sus procesos de enseñanza. Con respecto al esfuerzo de los docentes, también es relevante mencionar que la gestión de una enseñanza inclusiva debe ser integral y cooperativa entre todos los agentes educativos, el autor Canet (2009) menciona que: Para crear escuelas inclusivas se requiere de una respuesta educativa pertinente al alumnado en cuanto a sus necesidades, a las propuestas didácticas acorde a las características heterogéneas de sus alumnos, considerando la interacción entre comunidad educativa, el grado de coordinación y la utilización de los recursos y las prácticas educativas (p. 20).

- Las características de una educación inclusiva según Fierro \& Tapia (2012) Chaparro \& Caso (2012) CP Fierro (2013) son las siguientes;

- (a) el reconocer las necesidades de las personas y su propia atención frente a estas necesidades,

- (b) la valoración, evaluación y establecimiento de una atención inclusiva frente a las características de la persona (cultura, género, religión, entre otros),

- (c) trato equitativo,

- (d) promoción del sentido de pertenencia con la convivencia adecuada dentro de la comunidad educativa y

- (e) trabajo cooperativo.

- Por lo tanto, los principios fundamentales para determinar las prácticas pedagógicas que fomenten la educación inclusiva, desde:

- La Presencia: todos los alumnos están siempre presentes en todas las actividades, experiencias y situaciones de aprendizaje que se desarrollan en la 
escuela y en el aula. La Participación: todos los alumnos participan en todas las actividades, experiencias y situaciones, que se plantean en el aula y en el centro educativo como medio de aprendizaje de los alumnos.

- El Progreso: todos los alumnos progresan y aprenden con su participación en todas las actividades y situaciones que se crean y plantan en el aula y en el centro. (p. 67).

- Por consiguiente, la implementación de unas prácticas pedagógicas inclusivas no solo depende de las capacidades de los docentes desde sus procesos de enseñanza, sino que son un conjunto de acciones que cooperan e integran todos los agentes educativos. Con el fin de proporcionar un aprendizaje eficiente y adecuado para toda la comunidad, sin distinción ni diferencia entre los estudiantes (Carrillo. et.al, 2018, p.3).

\section{Avance de la educación inclusiva}

En los últimos años la educación inclusiva no avanza al ritmo deseable, lo que se atribuye, entre otros factores, a la existencia de una importante brecha entre el conocimiento que genera la investigación en educación inclusiva y el utilizado por el profesorado en su acción educativa.

Parece que en estos últimos años la educación inclusiva no avanza (Echeita, 2017) o al menos no lo hace al ritmo que sería deseable. Algunos autores indican al respecto que existe una brecha significativa entre el conocimiento generado por la investigación en educación inclusiva y el utilizado por el profesorado en su acción educativa (Korsgaard, Larsen, \& Wiberg, 2018; Marion \& Houlfort, 2015). Aunque se tienen todos los ingredientes para que la educación inclusiva sea una realidad en las escuelas, existe un exceso de teoría y un déficit en el desarrollo efectivo de este nuevo paradigma. Este desfase podría deberse a que la manera de comunicar los últimos avances y resultados de la investigación no sea la más adecuada. O tal vez a que el conocimiento generado por la investigación se aleja de los intereses y posibilidades de los centros educativos (Moliner García, Arnaiz Sánchez, Sanahuja Ribés, 2020, p 6).

\section{METODOLOGIA}

La investigación se desarrolló en la Ciudad de Pilar, y estuvo constituida por alumnos, directivos, equipo técnico del Ciclo y padres que tienen relación con los alumnos con necesidades específicas de apoyo educativo del Tercer Ciclo de la Educación Escolar 
Básica del Centro Regional de Educación “Mariscal Francisco Solano López” Pilar Paraguay, año 2020-2021,

Se efectúo una combinación de métodos cualitativos y cuantitativos.

Para la selección de la muestra se optó por un muestreo probabilístico intencional, ya que se conoce la población exacta elegidas para la investigación, con técnica de la encuesta.

\section{ANÁLISIS Y DISCUSIÓN}

Los resultados de las puntuaciones resultan de la siguiente sumatoria: Puntuación General: 19 preguntas por 8 encuestados (directores y equipo técnico) y 3 niveles de opción respuesta. Totalizando así: 456 puntos posibles, si es que todos optan por el nivel máximo 3, en su respuesta.

\section{Variable: Dimensión A: Crear CULTURAS inclusivas}

Para el efecto, se ha determinado establecer los siguientes subdimensiones: 1. Construir una comunidad y 2. Establecer valores inclusivos y en torno a ellos enunciaron 8 preguntas con sus 3 opciones de respuesta.

Figura 1. Dimensión A: Crear CULTURAS inclusivas- Subdimensiones: 1. Construir una comunidad y 2. Establecer valores inclusivos

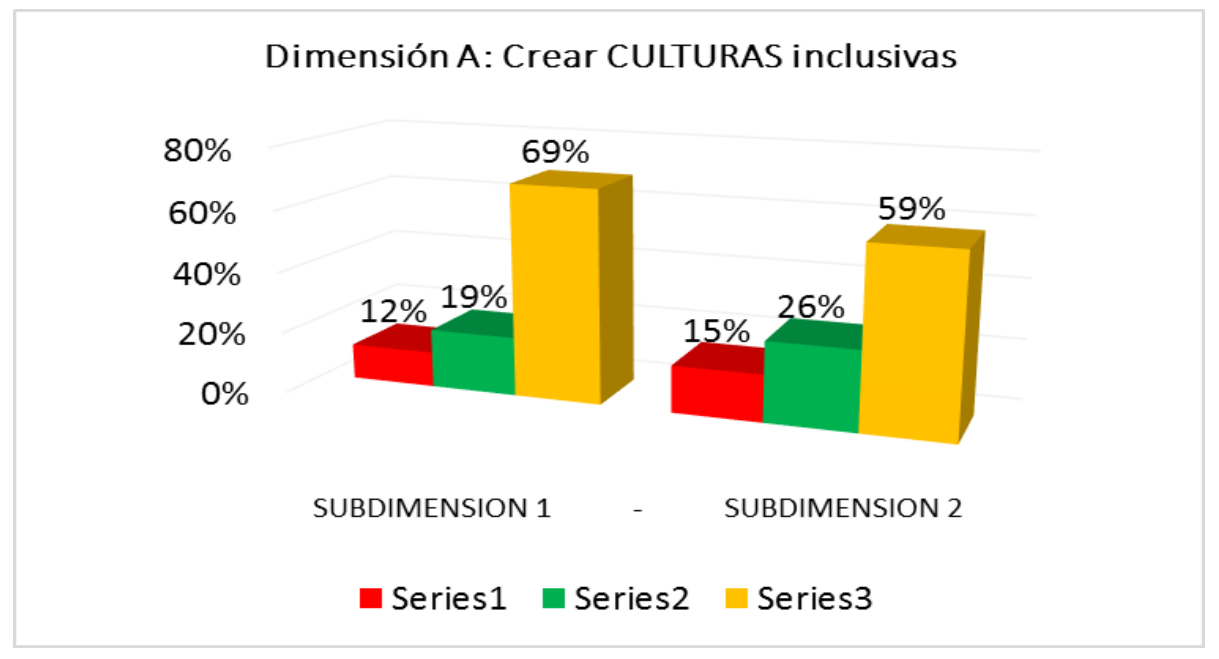

Interpretación Pedagógica. Luego de confrontar la distribución de los resultados numéricos de la dimensión 1. Construir una comunidad y 2. Sentido de acogimiento, con las interrogantes: Ayuda mutua entre alumnos, colaboración (docente-familia), respeto del profesores y alumnos, involucramiento en el centro, se tiene expectativas altas sobre todo el alumno, filosofía común entre la comunidad educativa, eliminación de barreras al aprendizaje de parte del profesorado. 
Estos datos resaltan se cumplen adecuadamente el contenido que suscribe a cada una de ellas, que el lenguaje es entendible y adecuado; establece valores inclusivos, los estudiantes se ayudan unos a otros, los miembros del personal de la escuela colaboran entre ellos, el personal de la escuela y el alumnado se tratan con respeto, no existen opiniones explícitas al respecto, por lo que se puede especular que los posibles desacuerdos radiquen en la falta de relación con la teoría, la no pertenencia a la sección o dimensión o la relevancia del mismo, existe relación entre el personal y las familias.

La no discriminación significa que todas las personas o grupos puedan acceder a cualquier nivel educativo y reciban una educación con similares estándares de calidad, que no se establezcan o mantengan sistemas educativos o instituciones separadas para personas o grupos y que no se inflija a determinadas personas o grupos un trato incompatible con la dignidad humana (Convención contra la discriminación en Educación, UNESCO, 1960).

\section{Variable: Dimensión B: Elaborar POLÍTICAS inclusivas}

Para el efecto, se ha determinado establecer los siguientes subdimensiones: 1. Desarrollar una escuela para todos y 2. Organizar el apoyo para atender a la diversidad Y en torno a ellos enunciaron 9 preguntas con sus 3 opciones de respuesta.

Figura $\mathbf{N}^{\circ}$ 2: Dimensión B: Elaborar POLÍTICAS inclusivas. Subdimensión 1. Desarrollar una escuela para todos y 2. Organizar el apoyo para atender a la diversidad

\section{Dimensión B: Elaborar POLÍTICAS inclusivas}

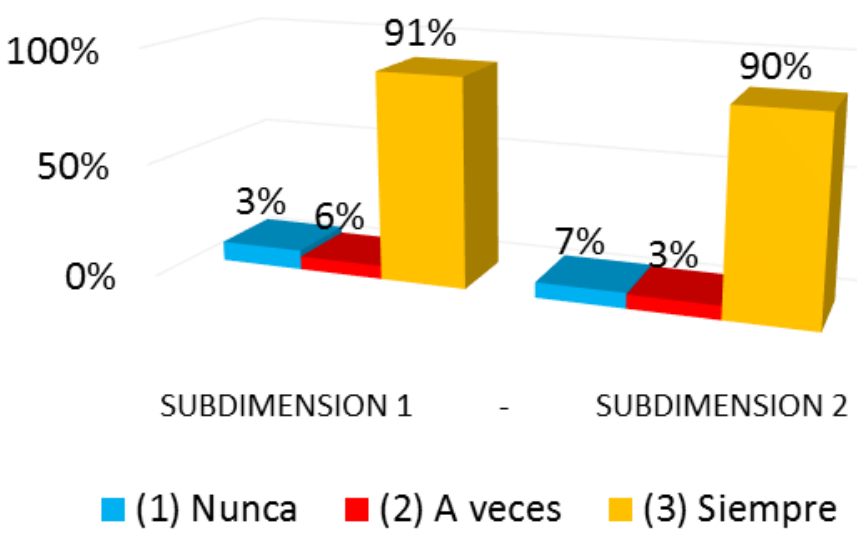


Interpretación Pedagógica. Luego de confrontar la distribución de los resultados numéricos de la dimensión, desarrollar una escuela para todos y organizar el apoyo para atender a la diversidad, queda distribuida de la siguiente manera:

Desarrollar una escuela para todos, los encuestados mencionan que entre las tenencias de $91 \%$ dicen siempre, el $6 \%$ a veces y el $9 \%$ nunca.

Organizar el apoyo para atender a la diversidad, los encuestados mencionan que entre las tenencias de.; $90 \%$ dicen siempre, el $63 \%$ a veces y el $7 \%$ nunca.

Dado los datos hacen resaltar que de cierta manera existen avance y cumplimiento de los derechos a la inclusión, constituidos por el avance en la consolidación de los derechos humanos, conocido como perspectiva ética, y el modelo social de discapacidad.

La educación al igual que la sociedad, ha reaccionado, hasta la introducción no muy lejana de medidas de democratización en las escuelas, de una forma muy parecida ante la diversidad humana: ha intentado reducir al mínimo los efectos de la misma basándose en distintas estructuras y reformas organizativas. Una breve mirada hacia el pasado común de distintos grupos en situación de exclusión escolar, podrá ayudarnos a construir comprehensivamente ese marco de análisis para entender la situación y retos más actuales del planteamiento inclusivo (Parrilla, 2002).

\section{Variable: Dimensión C: Desarrollar PRÁCTICAS inclusivas}

Para el efecto, se ha determinado establecer los siguientes subdimensiones: Subdimensiones:

1. Orquestar el proceso de aprendizaje -

2. Movilizar recursos. Y en torno a ellos enunciaron 12 preguntas con sus 3 opciones de respuesta. 
Figura N $\mathbf{N}^{\circ}$ 3: Dimensión C: Desarrollar PRÁCTICAS inclusivas -Subdimensiones: 1. Orquestar el proceso de aprendizaje -2. Movilizar recursos

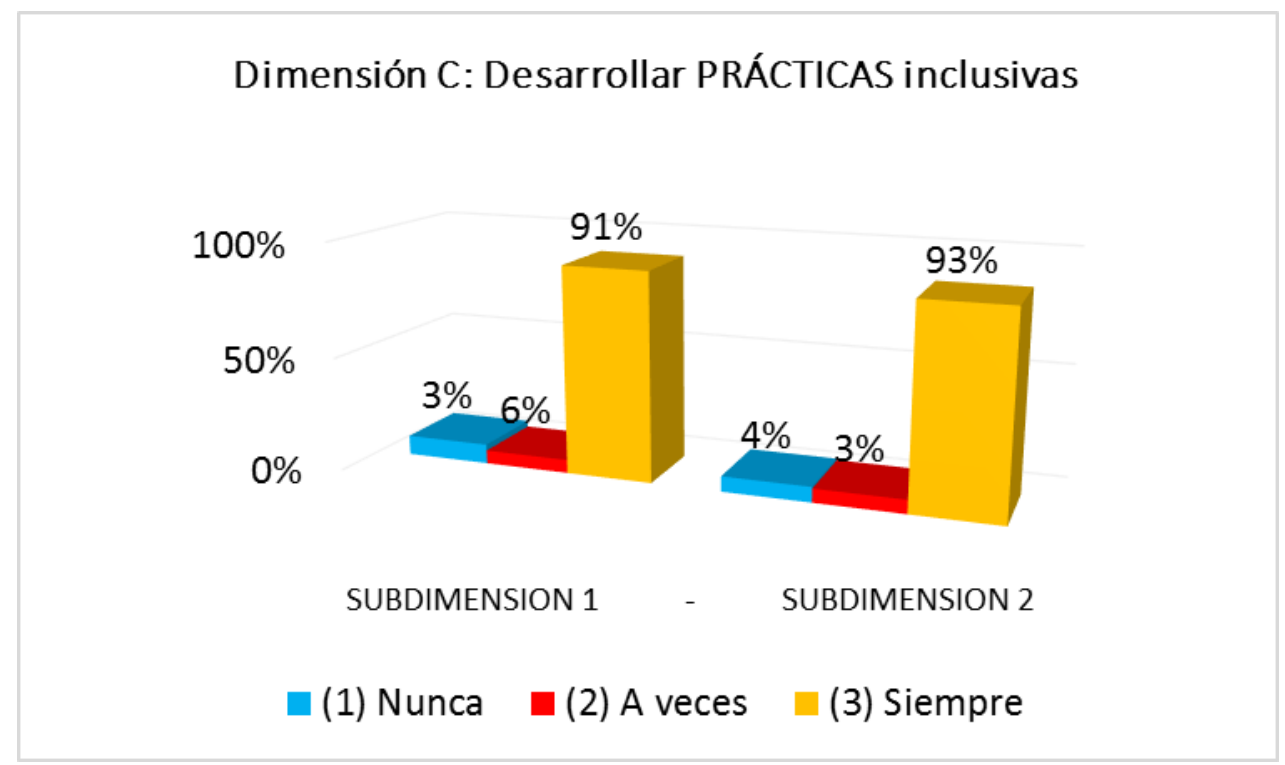

Interpretación Pedagógica. Luego de confrontar la distribución de los resultados numéricos de la dimensión 3 Evaluación, en esta parte se presentan los significados pedagógicos del análisis efectuado sobre la tabla 3.

En el Gráfico $\mathrm{N}^{\mathrm{o}} 3$ se observa la distribución de porcentajes de respuestas relativas a las dimensiones clasificado por los niveles de la escala de medición. Desarrollo de prácticas inclusivas alcanzándose el nivel más alto (Siempre), demostrando dos indicadores en los cuales se encontraron diferencias estadísticamente significativas, donde todo el alumnado participa en las actividades complementarias y extraescolares y los recursos de la escuela se distribuyen de forma justa para apoyar la inclusión.

\section{CONCLUSIONES}

La educación inclusiva está constituida por toda una serie de creencias y valores sociales, tales como: la aceptación de la diversidad como elemento enriquecedor de la comunidad educativa, la participación de todos los alumnos en el curriculum ordinario y en todas las actividades tanto escolares como extraescolares, la provisión a todos los estudiantes de iguales oportunidades para recibir servicios educativos efectivos, la atención diferencial y eficaz a la diversidad. Las escuelas inclusivas constituyen una aportación muy relevante en el contexto comunitario para conseguir una sociedad más igualitaria y menos excluyente. Los procesos de inclusión tienen que entenderse de forma multidimensional, es decir, hay que considerar el contexto social, político, 
económico y cultural para diseñar, desarrollar y poner en práctica la educación inclusiva, que trasciende la propia dimensión educativa (Dueñas Buey, 2010, p.3).

En gran medida, la práctica de la educación inclusiva está supeditada al contexto en el que se desenvuelve. Pero también con la inclusión se comparten una serie de valores comunes a todos los contextos en que se lleva a la práctica, es decir, en los diferentes países /contextos se dan elementos diferenciales que sin embargo confluyen en los valores subyacentes a las iniciativas y proyectos llevados a cabo (Dueñas Buey, 2010, p.3).

También, la Ley Nº5136 de Inclusión Educativa, garantiza la orientación, formación y/o capacitación adecuada y oportuna de los profesionales y demás integrantes de la comunidad educativa. (Art. $5^{\circ}$; inc. j)

Las directrices sobre políticas de inclusión en la educación, UNESCO (2009), explica que se hace necesario el enriquecimiento de la formación de quienes son responsables de la formación de individuos. Alegando que es reconocida la urgencia de incorporar nuevos elementos en la formación del profesorado, en todos los niveles, así como proveer alternativas para la formación continua que exige la Educación para Todos.

Si bien demuestran la mayoría de los encuestados, aceptación a los niños con NEE y la necesidad de inclusión de los mismos, no lo hacen por sentirse obligados por la Ley de Inclusión y por recibir sanciones en caso de incumplimiento de la Ley $\mathrm{N}^{\circ} 5136$; se ha observado un nivel alto del nivel de inclusión de los escolares con la participación de la comunidad educativa en estudio.

Las personas elaboran sus percepciones, a partir de sus experiencias en el ámbito personal y profesional. Estas influyen en la predisposición que se tiene para actuar de una manera u otro ante determinadas circunstancias, como por ejemplo la diversidad en el aula. Según la manera que una persona perciba la realidad, y de sus creencias y opiniones acerca de esta, dependerá su actitud hacia ella. Tanto las percepciones como las actitudes pueden manifestarse en las opiniones emitidas, ya que, las actitudes, así como las percepciones son componentes del desarrollo humano (Hurlock,1982).

Según, Papalia y Wendkos (1995) en el comportamiento humano confluyen las diferentes áreas del desarrollo: la percepción, en el ámbito del desarrollo cognitivo y las actitudes, en el área emocional- afectiva; las cuales se evidencian en las respuestas dadas por los encuestados. 
Se ha alcanzado un nivel máximo del (Nivel 3-Simrpe) donde los profesionales de la educación son partidarios de la inclusión educativa, manifiestan tener una buena actitud y expectativas positivas hacia sus alumnos, independientemente de cuáles sean sus características.

Tal y como reflejan los resultados, la dimensión cultura, políticas y prácticas han alcanzado valoraciones muy elevada se relaciona con la preparación necesaria y específica para el desarrollo de prácticas inclusivas, al destacar la importancia de crear una cultura escolar en la que los estudiantes se sientan competentes, valorados y no excluidos.

Para que el derecho a la educación sea garantizado con justicia éste tiene que ser reconocido y aplicado a todas las personas, sin excepción alguna, siendo obligación del Estado tomar las medidas necesarias para proteger al niño de toda forma de discriminación.

\section{REFERENCIAS BIBLIOGRAFICAS}

Ainscow, M. (2004). Desarrollo de Escuelas Inclusivas. Madrid, España: Narcea S. A. de Ediciones.

Ainscow, M. (2017). Haciendo que las escuelas sean más inclusivas: lecciones a partir del análisis de la investigación internacional. Revista de Educación inclusiva.

Arnaiz Sánchez, P. (2003). Educación inclusiva: una escuela para todos. Málaga. Aljibe.

Carrillo Sierra, S.M; et.al (2018). Prácticas Pedagógicas frente a la Educación Inclusiva desde la perspectiva del Docente. Revista Espacios. ISSN 07981015

Cuesta, M. (2009). Introducción al muestreo. Universidad de Ovideo.

Cultura, M. d. (2014). Decreto $N^{o} 2837 / 14$ Por la cual se Reglamente la Ley de Educación Inclusiva. Asunción .

Cultura, M. d. (2015). Resolución $N^{\circ} 01 / 15$ Que Regula el Régimen de Faltas y Sanciones . Asunción .

Dueñas Buey, M.L (2010). Educación Inclusiva. Revista Española de Orientación y Psicopedagogía, vol. 21, núm. 2, mayo-agosto, 2010, pp. 358-366 Asociación Española de Orientación y Psicopedagogía Madrid, España

Echeita, G. (2006). Educación para la Inclusión o Educación sin Exclusiones. Madrid: Narcea, S.A. De Edisiones. 
Escribano, G., \& Martínez C. (2013) Inclusión educativa y profesorado inclusivo. Madrid: Narcea S. A. De Ediciones.

Forgiony Santos, J. (2017). "Practicas Pedagógicas: concepciones, roles y métodos en la formación del psicólogo bolivariano", Practicas Pedagógicas, ISBN: 978980-402-237-1. Edit. Universidad del Zulia.

Hernández, R., Fernández, C. y Baptista, P. (2003). Metodología de la investigación (3 ed.). México: Editorial Mc Graw-Hill

Hernández, R., Fernández, C. y Baptista, P. (2008). Metodología de la investigación (3a ed.). México: Editorial Mc Graw-Hill

Ley $N^{o}$ 5136/13 De Educación Inclusiva. (2013). Asunción: Congreso Nacional del Paraguay.

Moliner García, O; Arnaiz Sánchez, P; Sanahuja Ribés, A (2020). Rompiendo la Brecha Entre Teoría y Práctica: ¿Qué Estrategias Utiliza el Profesorado Universitario para Movilizar? ¿El Conocimiento Sobre Educación Inclusiva? Educación XX1, vol. 23, núm. 1, 2020, -Junio, pp. 173-195 Universidad Nacional de Educación a Distancia España DOI: https://doi.org/10.5944/educXX1.23753

Organización de las Naciones Unidas para la Educación, la Ciencias y la Cultura. (2008). La Educación Inclusiva: El Camino hacia el Futuro. Ginebra: Centro Internacional de Conferencias .

Paraguay, C. N. (2001). Código No 1680/01de la Niñez y la Adolescencia . Asunción .

Rico, A. P. (2010). Políticas de Educación Inclusiva en América Latina. Revista Educación Inclusiva, Vol 3, N².

Sánchez, A. (2009). Políticas y prácticas educativas desde la perspectiva de la educación especial. Universidad de Almería. Documento Policopiado.

Stainback, S., \& Stainback, W. (2004). Aulas Inclusivas. Madrid, España: Narcea, S. A. De Ediciones.

Vaughan, M (2002). Índice de Inclusión - Desarrollando el aprendizaje y la participación en las escuelas, Centro de Estudio Inclusivos (SIE), Bristol UK 